\title{
L'Hermaphrodisme chez Bufo vulgaris
}

PAl:

\section{O. FUHRMANN}

Avec 6 figures dans le texte ${ }^{1}$.

L'hermaphrodisme est très rare chez les Urodèles, tandis que chez les Anours de nombreux cas ont été décrits par les auteurs. Ce phénomène semble ètre assez rare, malgré la trentaine de cas connus et signalés dans la littérature, si l'on considère le grand nombre de Rana qui sont disséquées chaque année dans les laboratoires de Zoologie.

L'hermaphrodisme est plus fréquent chez $B u f o$, bien que nous ne trouvions dans la littérature que fort peu d'indications ou de dessins de cette anomalie. Cependant cette anomalie présente un intérèt particulier à cause du singulier organe de Bidder placé en avant des glandes sexuelles mâles et femelles.

Spengel ${ }^{2}$ a observé deux cas d'hermaphrodisme chez Pelobates fuscus et Bufo cinereus = B. vulgaris). Chez Bufo vulgaris, les ovaires étaient bien développés, mais sans oviductes; ce cas ressemble donc probablement à notre fig. 4. Chez Pelobates fuscus, l'auteur trouva un hermaphrodite fort curieux qui

1 Je dois a mon ami, M. Th. Delachaux, artiste-peintre, l'exécution des dessins qui accompagnent ce travail.

2 Spengel, Das Urogenitalsystem der Amphibien. Arb. a. d. zool. zoot. Inst. Würzburg, 1876. - Zwitterbildung bei Amphibien, Biolog. Centralblatt, Bd. IV, 1884. 
montrait, en arrière du testicule, du còté droit, un petit ovaire avec des œufs normaux. Les canaux de MüLler faisaient défaut.

KNapPe ${ }^{1}$ en a observé une dizaine de cas dans ses recherches sur l'organe de Broper, mais il ne les a jamais décrits et ne donne qu'une petite figure d'un hermaphrodite pas très prononcé. Tous ces cas présentent des testicules bien développés et ont des ovaires à une ou plusieurs loges; l'auteur ne dit rien des oviductes. Ce qui est surtout curieux, c'est que, d'après Knappe, l'ovaire rudimentaire, ainsi que le testicule. possédaient partout leur organe de BIDDEr. Cette observation nous frappe d'autant plus que, dans les très nombreux cas que j’ai observés, je n'ai jamais vu aucune trace d'une disposition semblable. Je suis donc tenté de considérer l'interprétation de KNAPPE comme une erreur car, à en juger par son dessin fig. 1, pl. 28, les individus éludiés devaient ètre très petits et très jeunes, et de ce fait, la glande de BIDDEn et l'ovaire rudimentaire très semblables.

Ces derniers temps, Cerruti ${ }^{?}$ a décrit en détail deux cas intéressants, dont l'un ressemble au cas de notre fig. 2, avec cette différence que le testicule gauche manquait complètement dans la forme décrite par cet auteur. Le second hermaphrodite ressemble à notre fig. 4, mais les ovaires sont beaucoup moins développés que chez l'individu que nous décrivons plus loin.

En 1907, en disséquant une dizaine de Bufo vulgaris au laboratoire, nous trouvâmes 3 hermaphrodites dont un particulièrement intéressant parce que c'était l'hermaphrodite le plus parfait trouvé jusqu'alors chez les Amphibiens (voir fig. 6). Deux années plus tard, nous avons disséqué 91 mâles de Bufo et trouvé 17 hermaphrodites bien marqués, soit 18,6\%. En 1912, sur 72 Crapauds disséqués, 22 étaient hermaphrodites, donc $30,6 \%$. Tous ces matériaux viennent de la mème localité, soit d'un petit étang situé dans la forèt de Serroue, près de Neuchâtel, où se réunissent chaque année, au printemps, des cen-

1 Knappe, Das Biddersche Organ, Morphol. Jahrb., XI. Bd., 1886.

2 Cerroti, A. Sopra due casi di anomalie dell apparato riprodultore nel Bufo vulgaris. Anat. Anz., Bd. 30, 1907, p. 53-64 
taines de Crapauds. C'est au mois d'avril que les récoltes des Bufo furent faites.

Avant de grouper et de décrire les différents cas d'hermaphrodisme que nous avons constatés, nous tenons à donner une classification des hermaphrodites.

Geofrroy St-Hilaire, Meckel, J. Mülder, Hermann Klebs et Max Wевек ont essayé d'établir des classifications, mais c'est certainement celle de Stephan ${ }^{1}$ qui est la plus rationnelle.

Il distingue trois catégories d'hermaphrodisme :

Hermaphrodisme effectil.$\quad\left\{\begin{array}{l}\text { autogame. } \\ \text { réciproque. } \\ \text { successif. }\end{array}\right.$
Hermaphrodisme potentiel..$\left\{\begin{array}{l}\text { fécond. } \\ \text { stérile. }\end{array}\right.$
Hermaphrodisme rudimentaire $\left\{\begin{array}{l}\text { glandulaire. } \\ \text { tubulaire. } \\ \text { externe. }\end{array}\right.$

Ayant trouvé, chez Bufo, des cas d'hermaphrodisme rentrant dans chacune des grandes catégories distinguées par STEPHan, nous allons caractériser les groupes d'après les données de l'auteur. Stephas, dont l'étude très intéressante semble ètre peu connue, puisque nous n'avons trouvé de compte rendu ni dans le “Zoologisches Centralblatt», ni dans les “Jahresberichte de Naples ", appelle hermaphrodite effeclif l'individu capable de fonctionner comme mâle et comme femelle. "Dans le cas où il pourra féconder lui-mème les œufs qu'il aura produits, il sera autogame. L'hermaphrodisme réciproque est celui où deux individus hermaphrodites font un échange de leurs produits. Si l'animal est d'abord mâle puis femelle, l'hermaphrodisme sera successif. ” (Myxine.)

Dans le second groupe, celui des hermaphrodites potentiels, il existe toutes les parties qui seraient nécessaires pour que

1 Stephan, Pierre. De l'hermaphrodisme chez les Vertébrés, Thèse de la Faculté de Médecine de Montpellier, no 25, 1901. 
l'hermaphrodisme füt effectif; mais, pour certaines raisons, l'une de ces parties ne peut pas fonctionner et empèche l'animal de se comporter comme s'il appartenait aux deux sexes. Si l'incapacité fonctionnelle ne porte que sur un sexe, l'autre se comportera d'une façon normale; l'hermaphrodite sera fécond au mème titre qu'un individu unisexué normal. Si, au contraire, aucun des deux appareils ne fonctionne, l'hermaphrodite sera stérile.

Au groupe des formes avec hermaphrodisme rudimentaire, appartiennent tous les cas des limites de l'hermaphrodisme potentiel jusqu'aux premières traces de la bisexualité. Ce groupe est certainement le plus vaste et renferme la plus grande partie des cas d'hermaphrodisme connus.

Dans nos matériaux récoltés en disséquant 173 mâles de Crapauds (Bufo vulgaris), à l'époque de leur reproduction, nous avons rencontré des cas d'hermaphrodisme rentrant dans les groupes suivants :

1. Hermaphrodisme rudimentaire glandulaire (6 cas).

2. Hermaphrodisme rudimentaire tubulaire (3 cas).

3. Hermaphrodisme rudimentaire glandulaire et tubulaire (27 cas).

4. Hermaphrodisme potentiel fécond (2 cas).

5. Hermaphrodisme effecuif autogame (2 cas).

L'Hermaphrodisme rudimentaire glandulaire et tubulaire.

Le fait qu'il existe chez les mâles de Bufo vulgaris un organe de Brbder ayant une structure le rapprochant d'un ovaire et présentant la mème évolution et les mèmes phénomènes de dégénérescence, permet de considérer tous ces Crapauds mâles comme des animaux présentant normalement un hermaphrodisme rudimentaire glandulaire ${ }^{1}$. Stephax (loc. cit., p. 65 ) considère avec raison les Crapauds comme des Amphibiens avec une sorte d'hermaphrodisme rudimentaire dévié et régularisé.

\footnotetext{
1 Je tiens à signaler ici quelques cas où l'organe de Bidder, qui, chez la très grande majorité des Bufo vulgaris disséqués au mois d'avril, avait un diamètre de $4-6^{\mathrm{mm}}$, était rudimentaire ( 1 à $2 \mathrm{~mm}$ ) ou manquait mème complètement (1 cas).
} 
Nous ne voulons naturellement prendre ici en considération que les cas où il existe chez les mâles des canaux de MüLler ou un organe de Bidder présentant un développement anormal, c'est-à-dire montrant à son extrémité distale des formations qui ressemblent, comme aspect, à un ovaire plus ou moins développé. Si nous ne considérons que les cas d'hermaphrodisme rudimentaire, nous constatons que l'hermaphrodisme est rarement seulement glandulaire ou tubulaire, mais que le plus souvent les glandes ovariennes et les canaux de MüLter se présentent ensemble, sans qu'il y ait toujours une corrélation directe entre le développement des glandes et de leurs conduits. STEPHAN aurait donc dù ajouter dans son groupement des hermaphrodites rudimentaires (voir plus haut) le type de l'hermaphrodisme rudimentaire glandulaire et tubulaire.

Voici, du reste, le résumé des résultats de nos dissections en ce qui concerne l'hermaphrodisme rudimentaire :

En 1909, sur 91 Crapauds mâles, 16 étaient des hermaphrodites rudimentaires, tandis que sur 72 Bufo disséqués en 1912, 20 appartenaient à ce groupe d'hermaphrodites.

Ce qui nous frappe dans tous ces cas d'hermaphrodisme, c'est que les rudiments d'organes sexuels femelles ne sont presque jamais symétriquement développés, presque toujours, d'un còté ou de l'autre, l'ovaire ou le canal de Müller sont mieux différenciés.

Remarquons d'abord qu'assez souvent, sans qu'il y ait des traces d'hermaphrodisme, l'un des testicules est nettement plus petit que l'autre. Je signale ce fait parce que ce phénomène se présente également chez certains hermaphrodites, et on est alors facilement tenté de considérer cette réduction comme étant en corrélation avec le développement d'un ovaire accessoire, ce qui n'est certainement pas toujours le cas.

En général, il n'existe aucune trace de canaux de Mü LLER chez les mâles. Pourtant, dans un petit nombre de cas (6), nous en avons trouvé des rudiments très minimes qui semblaient sans relation avec le cloaque. En regardant de plus près, on voyait partir, en avant et en arrière, dans le mésentère qui portait ce 
court fragment de canal, une très fine ligne blanche, première ébauche d'un canal de Mürcer plus complet. Dans un mâle à glandes sexuelles normales, les deux canaux de Müller, bien que très courts, étaient contournés. Tous ces cas sont à considérer comme de légers cas d’hermaphrodisme tubulaire.

L'hermaphrodisme glandulaire apparaît presque toujours à l'extrémité distale de l'organe de BIDDEr et à son còté dorsal. L'ovaire plus ou moins développé du mâle hermaphrodite repousse alors en avant l'organe de BIDDEn, qui repose normalement sur l'extrémité antérieure du testicule. Les cas où le prolongement distal de l'organe de Broder qui, comme nous l'avons dit plus haut, a normalement un diametre transversal et longitudinal de 4-6 $\left.6^{\mathrm{mm}}\right)$, se trouve avoir absolument le mème aspect (transparence et couleur) et la mème structure que l'organe de Bidder, sont intéressants. Ainsi, nous voyons que dans 9 cas l'ovaire rudimentaire se prolonge en arrière en s'amincissant légèrement et atteint une longueur de $10^{\mathrm{mm}}$. Dans 3 de ces cas cette disposition était symétriquement développée, dans les autres seulement bien marquée d'un còté.

Dans la plupart des cas, lorsqu'entre l'organe de BıDoer et le testicule se glisse une néoformation produite par le premier, celle-ci a un aspect différent; elle est opaque et granuleuse (blanche dans le formol). Les 13 cas observés sont des variantes de celui représenté dans la fig. 1, ou le petit ovaire rudimentaire est inégalement développé sur les deux còtés.

Dans la fig. 1, l'organe de Bı⿻上丨 r droit, ainsi que l'extrémité antérieure du testicule gauche, semblent renfermer des œufs assez grands. L'ovaire rudimentaire est largement soudé avec la face dorsale de l'organe de Broder et les testicules. L'ovaire droit, ainsi que l'organe de BIDDER, renferment des traces de pigment noir. Le canal de Müllkr, très rudimentaire, n’est développé que du còté gauche; les glandes adipeuses, à droite surtout, sont énormes. Dix autres cas sont presque identiques a celui qui vient d'être écrit. L'organe de BIDDER, comme aspect et structure, est normalement conformé chez tous ces hermaphrodites et, ni lui, ni l'ovaire, ne montrent du pigment noir. Dans 
quelques cas, l'ovaire gauche devient plus apparent (jusqu'à $8^{\mathrm{mm}}$ de longueur) et les deux oviductes sont souvent développés sous forme de deux tubes courts et fins, non reliés au cloaque et allant à peine à mi-hauteur des testicules. Dans 2 cas, les deux ovaires, mieux développés, sont longs dę 7 à $10^{\mathrm{mm}}$ environ

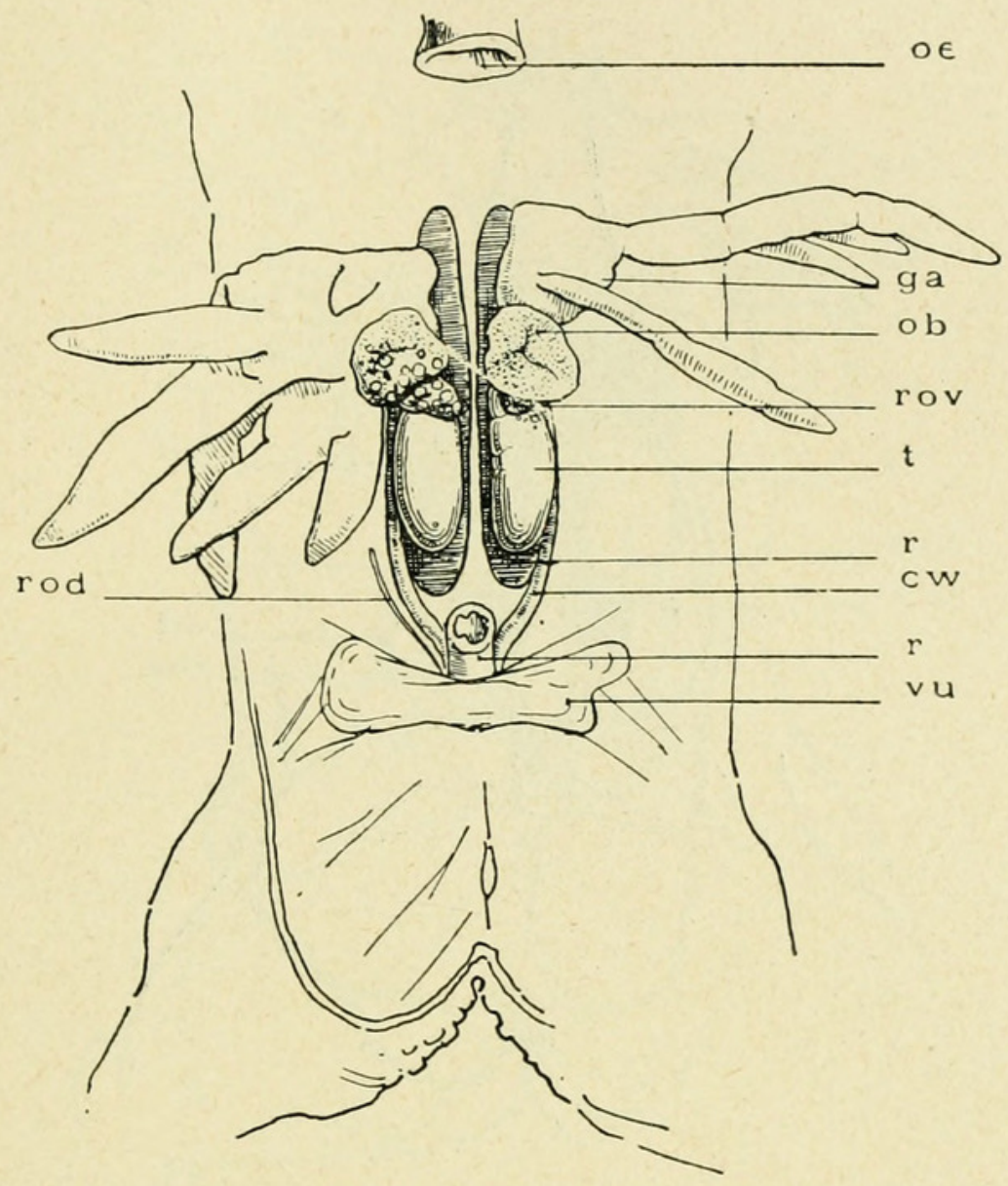

Fig. 1. - Hermaphrodite rudimentaire, glandulaire et tubulaire

$t$ testicule, $o b$ organe de Bidder, $c w$ canal de Wolff, rov ovaire rudimentaire, rod oviducte rudimentaire, ga glandes adipeuses, $r$ rein, $v u$ vessie urinaire, oe oesophage.

et larges de 2 à $3^{\mathrm{mm}}$, d'aspect opaque et granuleux; les oviductes font défaut (hermaphrodisme glandulaire).

Le cas de la fig. 2 diffère des précédents par le développement des oviductes qui sont parfaitement formés, mème avec la dilatation utérine. Les organes de Bidder sont normalement conformés; l'ovaire, qui se glisse entre cet organe et le testicule, est long de $6^{\mathrm{mm}}$ et large de 2 à $3^{\mathrm{mm}}$ sur le còté droit, tandis que, 
sur le còté gauche, la glande femelle est longue de $10^{\mathrm{mm}}$. Le testicule droit est long de $11^{\mathrm{mm}}$, le gauche n'est long que de $6^{\mathrm{mm}}$. Les glandes ovariennes diffèrent de celles des cas précédents par la présence de pigment noir.

Jusqu'à maintenant, l'ovaire était toujours peu développé,

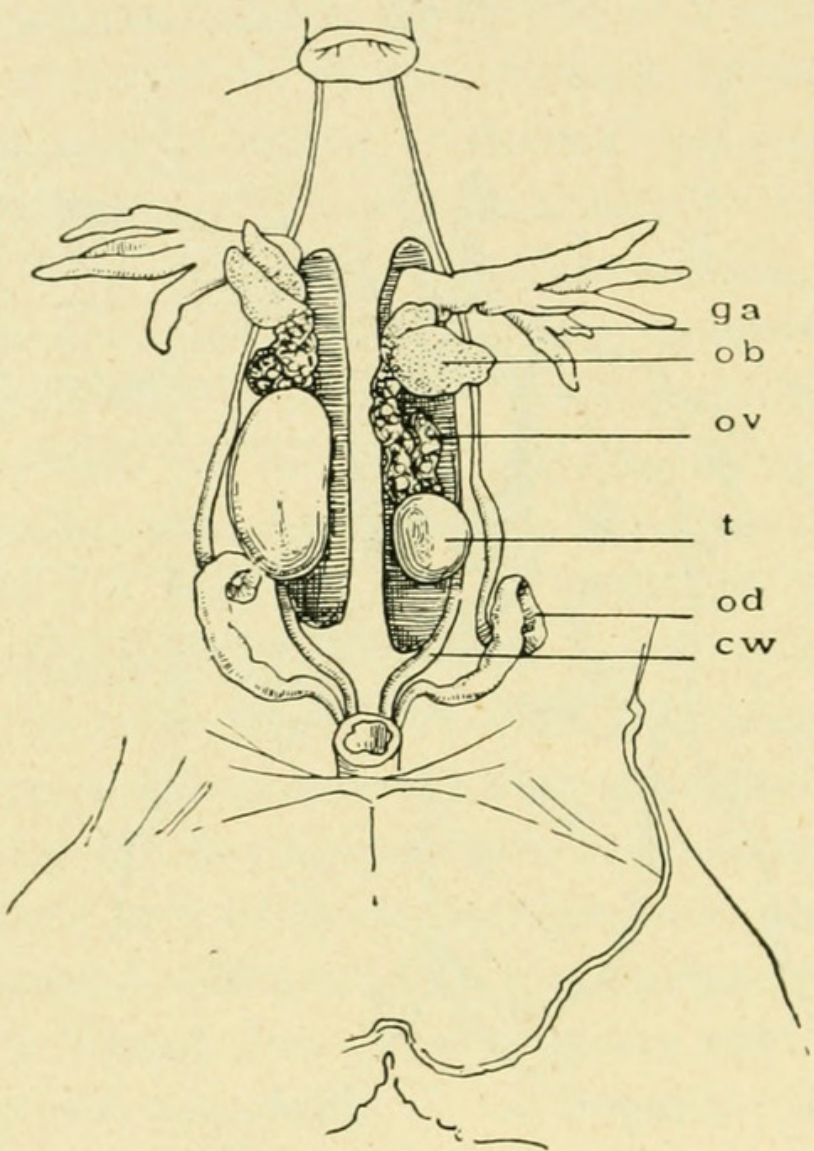

Fig. 2. - Hermaphrodite rudimentaire, glandulaire et tubulaire.

$t$ testicule, ob organe de Bidder, cw canal de Wolf, ov ovaire, od oviducte, ga glandes adipeuses.

plus étroit mème que la glande de Bidder et ne montrant aucune subdivision en poches ovariennes; dans les cas suivants, l'ovaire commence à prendre de plus en plus l'aspect d'une glande sexuelle femelle normalement conformée.

Dans 4 cas, les dispositions se trouvent ètre comme dans la fig. 3. La glande sexuelle, riche en pigment noir, est beaucoup plus petite à droite qu'à gauche. A gauche, l'ovaire est trilobé, 
long de $10^{\mathrm{mm}}$ et large de $9^{\mathrm{mm}}$. L'oviducte, à droite, est nul; à gauche il n'en existe qu'un rudiment de quelques millimètres. Les testicules sont normalement conformés, les glandes adipeuses énormes.

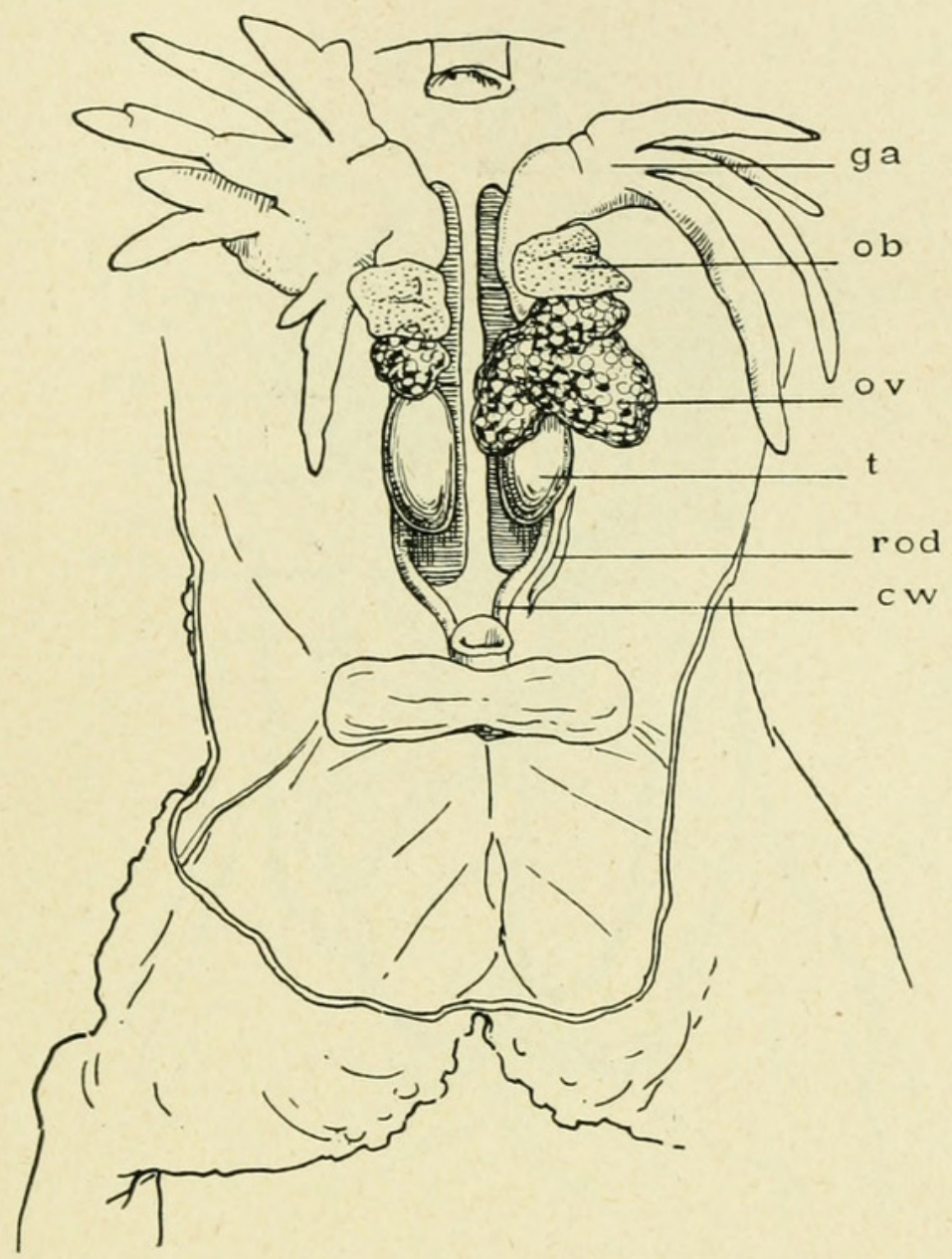

Fig. 3. - Hermaphrodite rudimentaire, glandulaire et tubulaire. $t$ testicule, $o b$ organe de Bidder, $c w$ canal de Wolf, ga glandes adipeuses, ov ovaire, rod oviducte rudimentaire,

Dans un cas l'ovaire ne renferme pas de pigment noir; dans deux autres cas les oviductes, bien que rudimentaires, sont un peu mieux développés que dans le cas de la fig. 3, atteignant l'extrémité antérieure des testicules. Dans un $4^{\mathrm{me}}$ cas, les ovaires pigmentés sont longs de $13^{\mathrm{mm}}$ à droite et de $10^{\mathrm{mm}}$ à gauche. 
L'oviducte droit manque, celui de gauche est comme dans la fig. 3 .

Chez un autre individu, l'ovaire droit, fortement lobé, est long de $6^{\mathrm{mm}}$ et large de $12^{\mathrm{mm}}$; à gauche, par contre, il est long de $17^{\mathrm{mm}}$, large de $12^{\mathrm{mm}}$ et également lobé 6 lobes); il couvre ainsi

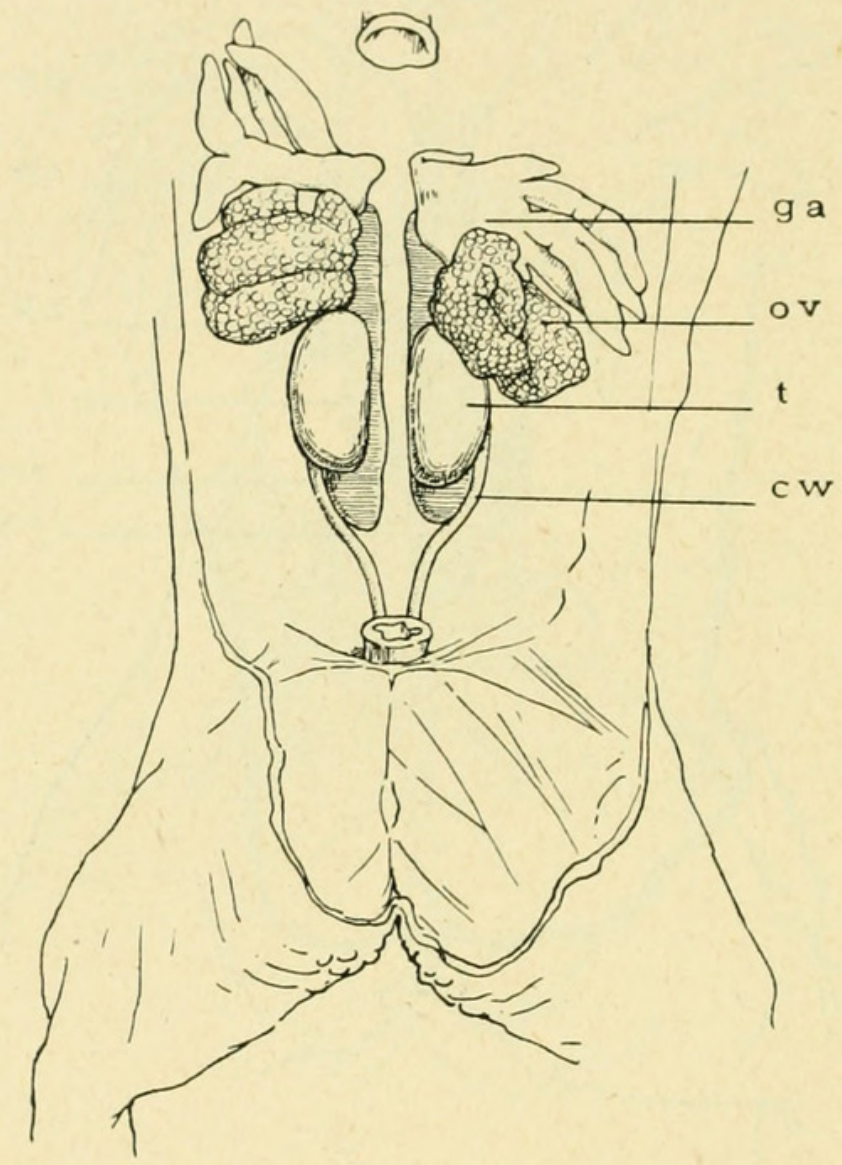

FIG. 4. - Hermaphrodite rudimentaire glandulaire.

$t$ testicule, $c w$ canal de Wolf, ov ovaire, ga glandes adipeuses.

le testicule, qui est fort réduit de ce côté, puisqu'il n'est long que de $5^{\mathrm{mm}}$ au lieu de $12^{\mathrm{mm}}$, comme sur le còté droit. Les oviductes très fins, fortement ondulés, vont jusqu'à la hauteur des organes de Bidder. Dans tous les cas précédents, l'ovaire est largement attaché au côté dorsal de l'organe de Bidper et fixé au testicule par son extrémité postérieure. 
Une disposition fort intéressante est celle qui est représentée dans la fig. 4 où les glandes de Bidder manquent complètement Les ovaires sont très bien développés, longs de 10 à $11^{\mathrm{mm}}$, larges de 9 à $10^{\mathrm{mm}}$; les oviductes font défaut hermaphrodite glandulaire).

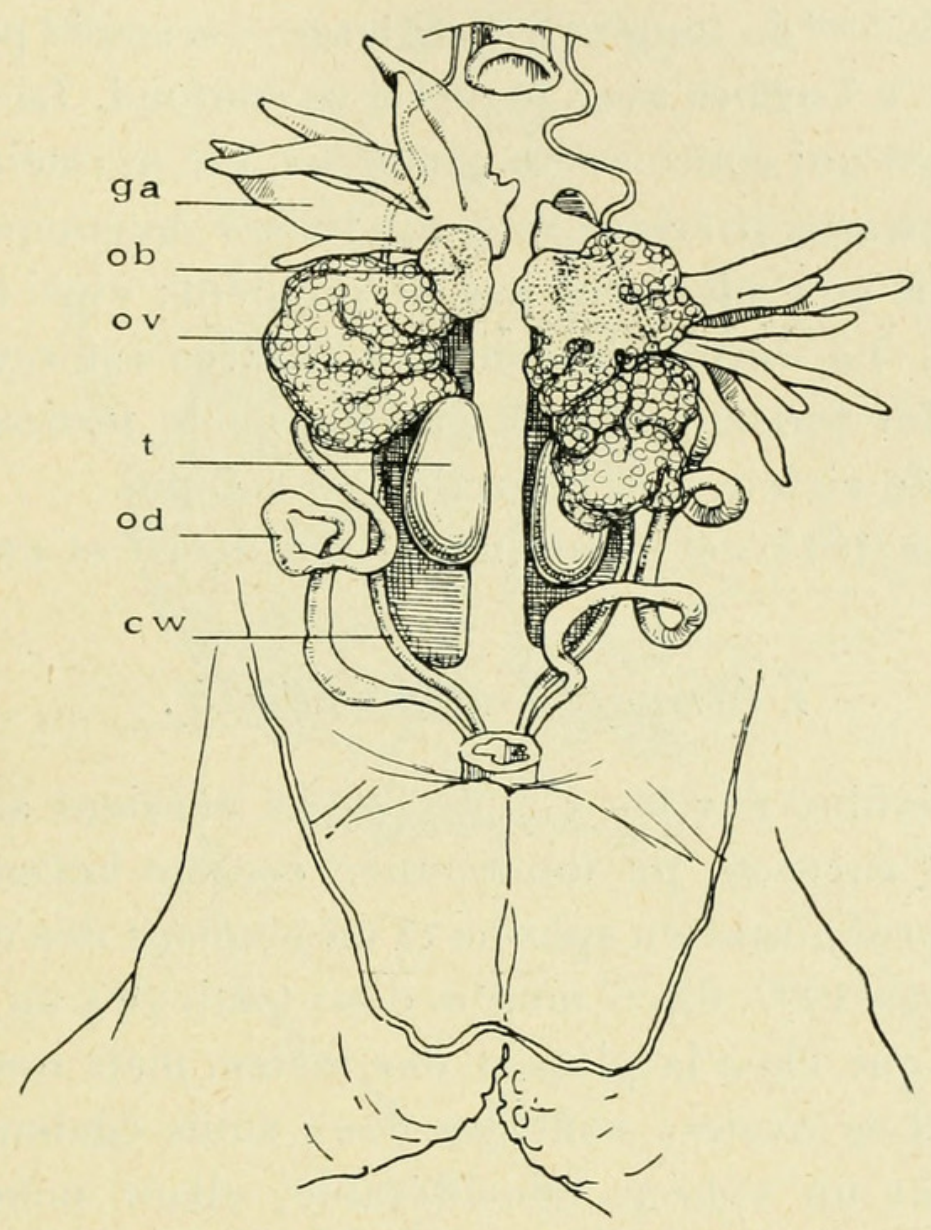

FIG. 5. - Hermaphrodite potentiel fécond.

$t$ testicule, ob organe de Bidder, cw canal de Wolf, ov ovaire, od oviducte, ga glandes adipeuses.

\section{L'hermaphrodisme potentiel.}

Sur les nombreux individus mâles de Bufo vulgaris, 2 seulement rentrent nettement dans cette catégorie d'hermaphrodites. La fig. 5 représente un des eas qui possèdent des oviductes bien différenciés, assez fortement contournés, certainement capables 
de fonctionner. L'ovaire droit est long de $13^{\mathrm{mm}}$ et large de $10^{\mathrm{mm}}$, avec un organe de Bidder large de $5^{\mathrm{mm}}$. Le testicule est long de $10^{\mathrm{mm}}$, et l'ovaire est largement soudé à son extrémité antérieure. L'ovaire gauche, également soudé au testicule, est long de $16^{\mathrm{mm}}$ et large de $10^{\mathrm{mm}}$; son extrémité postérieure recouvre plus de la moitié du testicule, qui est long de $7^{\mathrm{mm}}, 5$ seulement. L'organe de Bidper, de $4^{\mathrm{mm}}$ de long et $5^{\mathrm{mm}}$ de large, est soudé par toute sa face dorsale à l'ovaire avec lequel il se confond. La glande de Bidder, en ce qui concerne sa grandeur, est normalement développée; les sacs ovariens sont subdivisés en compartiments, mais les œufs ne renferment pas de pigment; ainsi les ovaires sont blancs. Le développement des ovaires est asymétrique, beaucoup plus fort sur le còté gauche, où le testicule semble avoir subi, de ce fait, une réduction de volume.

L’autre cas (1912) est identique à celui décrit et dessiné plus haut.

\section{L'hermaphrodisme effectif.}

Deux individus sur les 173 disséqués auraient sans doute, s'ils avaient survécu, pu fonctionner comme hermaphrodites parfaits en produisant du sperme et en pondant des œufs.

L'un des cas (1907, fig. 6) montre deux testicules un peu moins longs $\left(8^{\mathrm{mm}}\right)$ que chez la plupart des mâles, mais normalement conformés. Les ovaires sont des deux còtés également développés et ont un volume considérable, allant même un peu au-delà de l'extrémité distale des testicules. Leur longueur est la mème que celle des reins, et ils mesurent $2^{\mathrm{cm}}, 5$ de long et $1^{\mathrm{cm}}, 7$ de large. Les $œ u f s$ sont de taille et de couleur normales. L'organe de Bidder est intéressant parce qu'il n'est pas développé comme chez les mâles et les nombreux hermaphrodites étudiés, où il se présente toujours sous la forme d'un organe distinct nettement circonscrit excepté dans les cas représentés par les fig. 4 et 5). Dans notre cas, il est, des deux còtés, de petite taille et confondu avec l'ovaire; il a donc absolument l'aspect de l'organe de Bidder, que nous avons trouvé au mois d'avril) chez de rares femelles. Les oviductes sont très déve- 
loppés et fortement contournés. Dans leur région terminale, se dessine déjà la dilatation qui deviendra l'utérus renfermant les œufs immédiatement avant la ponte. L'extrémité proximale de l'oviducte portant l'entonnoir se trouve à còté du pharynx.

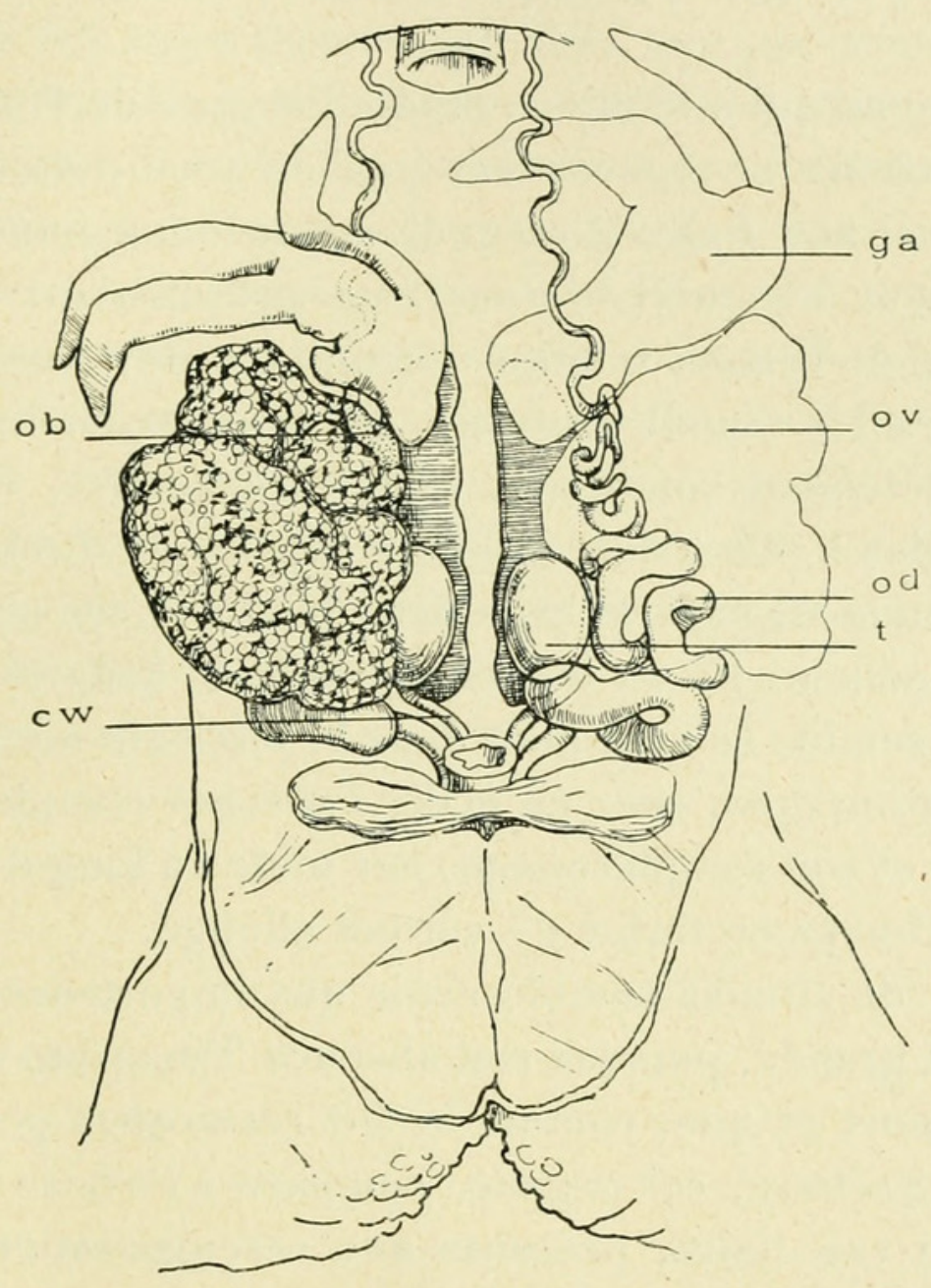

Fig. 6. - Hermaphrodite effectif autogame.

$t$ testicule, ob organe de Bidder. $c w$ canal de Wolf, ov ovaire, od oviducte, ga glandes adipeuses.

Les glandes adipeuses jaunes sont considérablement développées.

En 1912, nous avons trouvé également un exemplaire rentrant dans le groupe des hermaphrodites effectifs par le développement des glandes sexuelles. Les testicules étaient longs 
de $8^{\mathrm{mm}}$, les ovaires avaient une longueur de $23^{\mathrm{mm}}$ et une largeur de $16^{\mathrm{mm}}$. L'organe de Bidder, très petit, était confondu avec l'ovaire comme dans le $1^{\text {er }}$ cas. Les oviductes, par contre, étaient moins développés en ce qui concerne le diamètre et le nombre des circonvolutions. En effet, c'est sous l'aspect de l'oviducte de la fig. 5 que se présentait le canal de Mǘler.

C'est Knapp: qui a le mieux étudié l'organe de Bidper, mais ses observations ne concordent pas en tous points avec les nòtres. Ainsi nos Bufo, disséqués un ou deux jours après le réveil de leur sommeil hivernal, ne présentaient nullement des organes de BıDer ratatinés, mais bien développés, et cet organe, chez les femelles adultes qui le possédaient, se montrait complètement soudé aux glandes sexuelles, et non pas séparé d'elles ${ }^{1}$. Chez les mâles, l'organe de Bipder est en général nettement séparé, mais dans bien des cas nous l'avons trouvé intimément lié au testicule et mème, dans certains cas, cet organe semble se continuer dans le testicule mème.

Stephan remarqua, chez un mâle, l'absence complete du testicule droit et son remplacement, sur toute sa longueur, par un organe de Bidder en forme de cordon allongé.

L'organe de Bidner semble avoir une importance physiologique assez grande, puisque son absence (Policard) est bientòt suivie de mort et que, comme le fait remarquer pour la première fois Stephan, cet organe, comparé à l'ovaire qui est en somme peu vasculaire, présente une vascularisation extrême. Nous ignorons encore complètement quelle peut être la fonction de cet organe, mais l'étude de son évolution, de sa structure, de sa régression et régénération, a montré nettement que sa valeur morphologique doit ètre celle d'un ovaire rudimentaire.

Notre étude des nombreux cas d'hermaphrodisme avec tous les intermédiaires depuis le Crapaud mâle à organe de Bidder

1 Stephan (loc. cit.) dit qu'au point de vue histologique, le passage de l'un à l'autre se fait progressivement, et sur les coupes on ne voit pas de limite bien tranchée. 
normalement conformé, jusqu'aux formes d'hermaphrodites parfaits et au cas où l'organe de Bidder manque complètement et se trouve remplacé par un ovaire bien développé, confirment l'opinion des auteurs qui considèrent cet organe comme un ovaire rudimentaire et les Crapauds comme des animaux à hermaphrodisme rudimentaire.

Un autre fait assez intéressant, bien que pas nouveau, est la constatation qu'il n'existe aucune corrélation directe entre le développement des glandes ovariennes des hermaphrodites et la différenciation de leurs canaux de Müller. Tantòt les ovaires sont très bien développés et les canaux de MüLler très rudimentaires ou même nuls, tantòt dans d'autres cas plus rares il est vrai, les canaux de Müller se trouvent mieux développés que l'ovaire rudimentaire. C'est dans un nombre de cas restreint seulement que nous avons trouvé, chez les hermaphrodites, une harmonie parfaite entre le développement des glandes sexuelles femelles et leurs conduits. 


\section{$2 \mathrm{BHL}$ Biodiversity Heritage Library}

Fuhrmann, Otto. 1913. "L'hermaphrodisme chez Bufo vulgaris." Revue suisse de zoologie 21, 331-345. https://doi.org/10.5962/bhl.part.75226.

View This Item Online: https://www.biodiversitylibrary.org/item/40668

DOI: https://doi.org/10.5962/bhl.part.75226

Permalink: https://www.biodiversitylibrary.org/partpdf/75226

\section{Holding Institution}

MBLWHOI Library

\section{Sponsored by}

MBLWHOI Library

\section{Copyright \& Reuse}

Copyright Status: NOT_IN_COPYRIGHT

This document was created from content at the Biodiversity Heritage Library, the world's largest open access digital library for biodiversity literature and archives. Visit BHL at https://www.biodiversitylibrary.org. 